\title{
Infarto renal agudo
}

\author{
Agustín Santamaría Marína, Carmen Monroy Gómez ${ }^{b}$, Angela Garcia- Minguillán Talaverab, \\ Teresa Sánchez- Maroto Lozano ${ }^{b}$
}

\begin{abstract}
a Servicio de Urgencias. Hospital General Universitario de Ciudad Real.

${ }^{b}$ Servicio de Medicina Interna. Hospital General Universitario de Ciudad Real.

Correspondencia: Agustín Santamaría Martín c/ Obispo

Rafael Torija s/n. CP: 13005. Ciudad Real. Dirección postal: c/ Hernán Pérez del Pulgar, 4, $2^{\circ}$ D. CP: 13001. Ciudad Real. Email:

asantamariamarin@hotmail.com
\end{abstract}

Recibido el 8 de marzo de 2013 Aceptado para su publicación 25 de abril de 2013

\section{RESUMEN}

El infarto renal es una causa infrecuente de dolor abdominal. Se debe sospechar en todo paciente con antecedentes de arritmias cardíacas o enfermedad valvular que acudan al servicio de urgencias por dolor abdominal agudo y que además presenten elevación de la lactato - deshidrogenasa (LDH) y alteración en el sedimento urinario. En todos estos casos el procedimiento diagnóstico de elección es la tomografía axial computerizada (TAC) abdominal con contraste. Presentamos el caso clínico de una mujer de 78 años con fibrilación auricular (FA) permanente con niveles infraterapéuticos de acenocumarol y antecedente de una isquemia arterial aguda en miembro inferior que acudió al servicio de urgencias por dolor abdominal.

PALABRAS CLAVE: Infarto renal, Dolor abdominal, Embolia, Fibrilación auricular.

\section{ABSTRACT}

Renal infarction is a frequent cause of stomach pain. It should be suspected in all patients with with a history of cardiac arrhythmias or heart valve disease going to the casualty department (emergency room) for acute stomach pain and who also show signs of lactate elevation dehyrogenase (LDH) and urine sediment disorder. In all these cases, the diagnostic procedure of choice is abdominal computerized tomography (CT) with contrast. We report the clinical case of a 78 year old woman with permanent atrial fibrillation (AF) with infra-therapeutic levels of acenocoumarol and history of a lower limb arterial ischaemia who went to the casualty department because of stomach pain.

KEYWORDS: Renal infarction. Stomach pain. Embolism. Atrial fibrillation.

\section{INTRODUCCIÓN}

El infarto renal se produce como consecuencia de la obstrucción aguda de una o más de las ramas de las arterias renales principales.

Es una causa poco frecuente de dolor abdominal, con una incidencia del $1,4 \%$, siendo una entidad infradiagnosticada ${ }^{1,2}$. Esto es debido a que existe un aumento progresivo de la población mayor de 65 años y una alta prevalencia de enfermedad cardiovascular en la población general ${ }^{3}$. Es más frecuente en la mujer durante la $6^{a}$ década de la vida.

La etiología más frecuente es la embólica, seguida de la trombosis. Otras causas son los traumatismos previos, los estados de hipercoagulabilidad, las anomalías vasculares y las drogas ${ }^{1,4,5}$.

La sintomatología es bastante inespecífica, lo cual dificulta el diagnóstico precoz ${ }^{1}$.

El tratamiento se basa en la anticoagulación, la fibrinólisis y la cirugía de revascularización ${ }^{5}$.

\section{OBSERVACIONES CLÍNICAS}

Mujer de 78 años que acudió al servicio de urgencias por dolor en flanco 
izquierdo irradiado a espalda de 48 horas de evolución. Se acompañaba de vómitos y debilidad generalizada. Además, refería que hacía 3-4 días había presentado un episodio autolimitado de dolor, parestesias y frialdad en muslo izquierdo.

Entre sus antecedentes se incluían alergia a los contrastes yodados, hipertensión arterial (HTA) diabetes mellitus tipo 2, dislipemia y FA. Seguía tratamiento con digoxina, acenocumarol, insulina, metformina, irbesartan y amlodipino.

En la exploración física destacaba presión arterial de 197/56 mmHg, frecuencia cardíaca de $95 \mathrm{lpm}$, temperatura de $37,8^{\circ} \mathrm{C}$, deshidratación leve y abdomen doloroso principalmente en fosa renal izquierda y flanco izquierdo.

En las pruebas complementarias realizadas se evidenció leucocitos 22.900 miles/ $\mu$ l (segmentados $84 \%$ y linfocitos $6,5 \%$ ), urea $115 \mathrm{mg} / \mathrm{dl}$, creatinina $3,1 \mathrm{mg} / \mathrm{dl}$, GOT $175 \mathrm{UI} / \mathrm{L}$, GPT $155 \mathrm{UI} / \mathrm{L}$, LDH $2368 \mathrm{UI} / \mathrm{L}, \mathrm{PCR}>20 \mathrm{mg} / \mathrm{dl}$, INR 1.8, hematuria y proteinuria. En el electrocardiograma se apreciaba una fibrilación auricular con respuesta ventricular aproximada de $80 \mathrm{lpm}$. En la ecografía abdominal llamaba la atención un riñón izquierdo de $137 \mathrm{~mm}$, con papilas edematosas, cortical con zonas hiperecogénicas, mala diferenciación corticomedular, así como mínima cantidad de líquido libre perirrenal (figura 1).

Ante la sospecha de isquemia renal de origen cardioembólico, se consultó con el servicio de nefrología que inició tratamiento con heparina

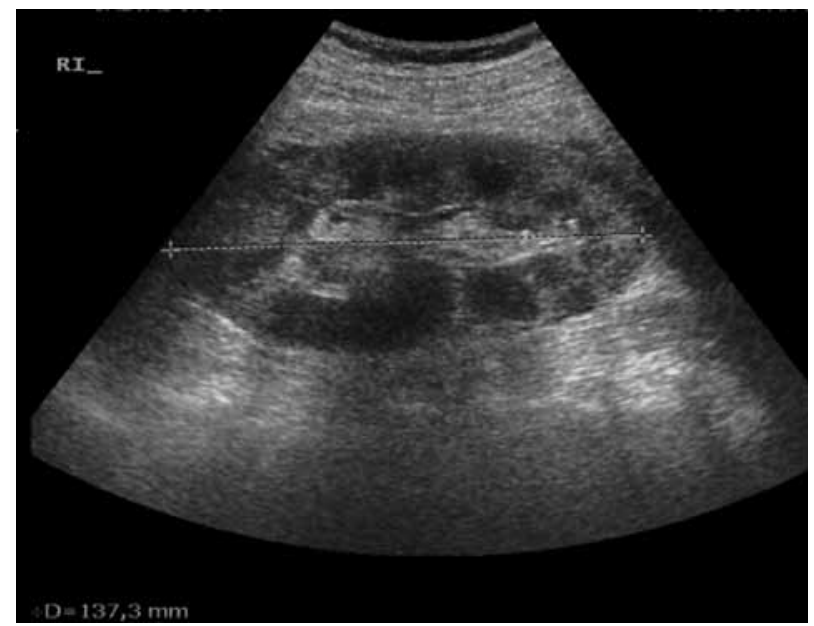

Figura 1: Ecografía renal realizada en urgencias en la que se observa un riñón aumentado de tamaño, con papilas edematosas, cortical con zonas hiperecogénicas y liquido libre perirrenal, todo ello compatible con nefropatía aguda. de bajo peso molecular a dosis terapéuticas tras desestimar tratamiento quirúrgico y fibrinolítico.

Una vez en planta previa premedicación con corticoides, dexclorfeniramina y acetilcisteína, se realizó ecodoppler renal con contraste en el que se objetivaron múltiples cuñas hipocaptantes sugerentes de infartos renales izquierdos.

La paciente evolucionó favorablemente, siendo dada de alta a los 10 días con mejoría progresiva de la función renal (creatinina al alta $2.3 \mathrm{mg} / \mathrm{dl}$ ), mejor control de las cifras de presión arterial y sin nuevos eventos embolígenos.

\section{DISCUSIÓN}

El infarto renal es una entidad clínica de difícil diagnostico debido a que la sintomatología que produce es muy inespecífica. La forma más frecuente de presentación suele ser el dolor abdominal que puede ser difuso, localizado en flanco o en región lumbar. Se puede acompañar de nauseas, vómitos, fiebre, HTA, hematuria y oligoanuria ${ }^{1,2,5}$.

Esta clínica nos obliga a realizar diagnóstico diferencial con otras patologías como el cólico renal, la pielonefritis, la isquemia intestinal, la colecistitis y la pancreatitis ${ }^{6,7}$.

En las pruebas de laboratorio podemos encontrar leucocitosis con neutrofilia, aumento LDH y creatinina, hematuria y proteinuria ${ }^{1,3}$. La elevación de LDH es el hallazgo más sensible, presente en casi el $100 \%$ de los casos, aunque es bastante inespecífico ${ }^{5}$. Se empieza a elevar a partir de las primeras 12-24 horas. El fracaso renal agudo, suele aparecer en aquellas ocasiones en las que el infarto es bilateral (15-30\%), o bien cuando existen microembolias en el riñón contralateral o en el paciente con riñón único funcionante.

El caso de nuestra paciente sugiere un infarto renal de causa embolígena por tratarse de una FA con niveles infraterapéuticos de acenocumarol y presentar clínica sugestiva, episodio embolígeno previo, HTA mantenida y elevación de LDH en la analítica.

El diagnóstico se realiza con ecografía, TAC con contraste, ecodoppler, gammagrafía o arteriografía. La ecografía abdominal con una sensibilidad aproximada del $11 \%$ es de poca utilidad. Con la TAC, la sensibilidad se incrementa hasta un $80 \%$, ya que se ponen de manifiesto defectos de perfusión en el parénquima renal con forma de 
cuña, con límites netos y rectilíneos sugerentes de infarto reciente. Sin embargo la arteriografía es la prueba más sensible y específica pero al tratarse de un procedimiento agresivo no es de elección ${ }^{1,5}$. Por este motivo, la TAC de abdomen con contraste al ser un método poco inocuo y no invasivo se ha convertido en el procedimiento diagnóstico de referencia ${ }^{2,5,6}$.

El tratamiento es bastante controvertido y se basa en la anticoagulación, la fibrinólisis y la cirugía de revascularización ${ }^{1,3,5}$. La fibrinólisis sólo sería útil si se aplica en las primeras 3 horas, que es cuando el tejido isquémico aún es viable. La cirugía de revascularización estaría indicada en los casos de origen traumático, en los infartos renales bilaterales o en los que ocurren en pacientes monorrenos o con riñón único funcionante. En el resto de los casos el tratamiento se basa en la anticoagulación. Está también está indicada como tratamiento profiláctico para prevenir nuevos eventos, por ello siempre se debe asociar a las diferentes opciones terapéuticas 5 .

En nuestro caso, el tratamiento estuvo condicionado por el tiempo de evolución del cuadro (se realizó a las 48 horas del inicio de la clínica). La fibrinólisis es el tratamiento de elección en los casos precoces, que en la práctica clínica son una minoría. Por tanto, el tratamiento de elección en nuestra paciente fue la anticoagulación.

En conclusión, ante un paciente con dolor abdominal, elevación de LDH, alteraciones en el sedimento urinario y alto riesgo de tromboembolia debemos sospechar esta entidad.

\section{BIBLIOGRAFÍA}

1. Hazanov N, Somin M, Attali M, Beilinson N, Thaler M, Mouallen $\mathrm{M}$ et al. Acute Renal artery embolism. Fortyfour cases of renal infarction in patients with atrial fibrillation. Medicine 2004; 83:292-299.

2. Korzets Z, Plotkin E, Bernheim J, Rivka Z. The clinical spectrum of acute renal infarction. Isr Med Assoc J 2002, 4: 781-784.

3. Gómez Ara AM, García García A, Cruz López A, Burillo Fuentes P. Infarto renal como causa infradiagnosticada de dolor abdominal. Emergencias 2010; 22: 117-119.

4. Ruíz Hernández JJ, Suárez Ortega S, Rodríguez Otero F, Pardo Moreno MD, Arkuch Saade ME y Palop Cubillo L. Infarto renal agudo de origen embólico en paciente anciano en fibrilación auricular. Rev Esp Geriatric Gerontol. 2006; 41(2): 134-7.

5. Xambre L, Cerqueira M, Silva V, Almeida M, Prisco R, Carreira $F$ et al. Isquemia renal aguda - causa rara de lumbalgia. Actas Urol Esp 2005;29 (3): 322-331.

6. Elalouf V, Vandwalle L, Viart L, Petit J, Saint F. La colique néphrétique ischémique de l'infarctus rénal: plaidoyer pour la tomdensitométrie hélicoïdale systématique en urgence. Progrès en urologie (2011) 21, 184-190.

7. Barbagelata López A, Lado Lema P, Lorenzo J, Barbagelata López C, Rodríguez Alonso A, Suárez Pascual $\mathrm{G}$ et al. El infarto renal en la valoración de dolores lumbares. Arch Esp Urol. Vol. 61, n 5, 2008, pp 646-649. 